\title{
Neural Dynamics in Monkey Parietal Reach Region Reflect Context-Specific Sensorimotor Transformations
}

\author{
Alexander Gail ${ }^{1,2}$ and Richard A. Andersen ${ }^{2}$ \\ ${ }^{1}$ Bernstein Center for Computational Neuroscience Göttingen, German Primate Center, 37077 Göttingen, Germany, and ${ }^{2}$ Division of Biology, California \\ Institute of Technology, Pasadena, California 91125
}

\begin{abstract}
We investigated the neural dynamics of sensorimotor transformations in the parietal reach region (PRR) of monkeys. To dissociate sensory from motor goal representations, we used a memory-guided anti-reach task. The monkeys had to reach either to a visually instructed, memorized peripheral target position (pro-reach) or to a diametrically opposed position (anti) while keeping central ocular fixation. Pro- and anti-reaches were randomly interleaved and indicated by a color instruction from the beginning of each trial. We analyzed spatiotemporal single-cell tuning and performed time-resolved population decoding to quantify the dynamic representation of the spatial visual cue, the reach goal, and the currently valid task rule (pro/anti mapping). Sensory information regarding the visual cue position was represented weakly during a short period of cue visibility. PRR predominantly encoded the reach goal from the end of the cue period on. The representation of the reach goal in the memory task evolves later for the anti-compared with pro-reaches, consistent with a 40-50 ms difference in reaction time between the two task rules. The task rule could be decoded before the appearance of the spatial cue, which indicates that abstract rule information is present in PRR that is independent of spatial cue or motor goal representations. Our findings support the hypothesis that PRR immediately translates current sensory information into reach movement plans, rather than storing the memorized cue location in the instructed-delay task. This finding indicates that PRR represents integrated knowledge on spatial sensory information combined with abstract behavioral rules to encode the desired movement goal.
\end{abstract}

Key words: sensorimotor transformation; posterior parietal cortex; motor intention; goal-directed behavior; anti-reach; S-R compatibility

\section{Introduction}

Identical external sensory information can be used to plan and guide different movements depending on internal behavioral goals or imposed task rules. We can guide our reach to a glass, if we want to drink, or a bottle, if we want to refill the glass. Reach movement plans depend not only on the location and shape of the target object but also on more abstract goals. Goal-directed behavior requires the integration of rules into sensorimotor transformations.

Areas in the superior lobe of the posterior parietal cortex (PPC), as part of a complex, reciprocally connected frontoparietal network, have been shown to contribute to skeletomotor sensorimotor transformation processes (for review, see Kalaska, 1996; Wise et al., 1997; Burnod et al., 1999; Battaglia-Mayer et al., 2000; Andersen and Buneo, 2002; Buneo and Andersen, 2006). The parietal reach region (PRR) in the medial intraparietal sulcus of the PPC is especially involved in the planning of reach move-

Received April 11, 2006; revised July 26, 2006; accepted July 27, 2006.

This work was supported by the National Institutes of Health, Defense Advanced Research Projects Agency, and Office of Naval Research. We thank Axel Lindner, Marina Brozovic, and Sam Musallam for valuable comments on a previous draft of this manuscript, K. Pejsa, N. Sammons, and L. Martel for animal care, and T. Yao and V. Shcherbatyuk for administrative and technical support.

Correspondence should be addressed to Dr. Richard A. Andersen, Division of Biology, California Institute of Technology, M/C 216-76, 1200 East California Boulevard, Pasadena, CA 91125. E-mail: andersen@vis.caltech.edu. DOI:10.1523/JNEUROSCI.1570-06.2006

Copyright $\odot 2006$ Society for Neuroscience $\quad$ 0270-6474/06/269376-09\$15.00/0 ments (Mountcastle et al., 1975; Johnson et al., 1996; Snyder et al., 1997; Marconi et al., 2001; Battaglia-Mayer et al., 2003) with spatial reach goals being mainly represented in visual coordinates (Batista et al., 1999; Buneo et al., 2002). The neural representations of abstract behavioral rules, and processes related to learning them, are typically associated with frontal (sub)cortical networks, including prefrontal cortex, dorsal premotor cortex, and basal ganglia (Petrides, 1982; Toni and Passingham, 1999; White and Wise, 1999; Wallis et al., 2001; Wallis and Miller, 2003; Nixon et al., 2004; Boettiger and D'Esposito, 2005; Pasupathy and Miller, 2005; Petrides, 2005). Other studies also suggest a role of PPC in representing abstract rules (Stoet and Snyder, 2004), especially once they are well established (Grol et al., 2006). This raises the questions how abstract rules are integrated into sensorimotor transformation processes. How and where are multiple context-specific visuomotor mappings for visually guided reaches represented?

We used a memory-guided anti-reach task to investigate ruledependent sensorimotor mapping in PRR of monkeys. The monkeys had to perform two alternative spatial visuomotor transformations, either to reach toward the memorized location of a previously flashed visual cue, or to a location diametrically opposite of it. The spatial dissociation of the cue and motor goal in this task (Georgopoulos et al., 1989; Boussaoud and Wise, 1993a,b; di Pellegrino and Wise, 1993; Funahashi et al., 1993; Crammond and Kalaska, 1994; Schlag-Rey et al., 1997; Zhang et al., 1997; 
A

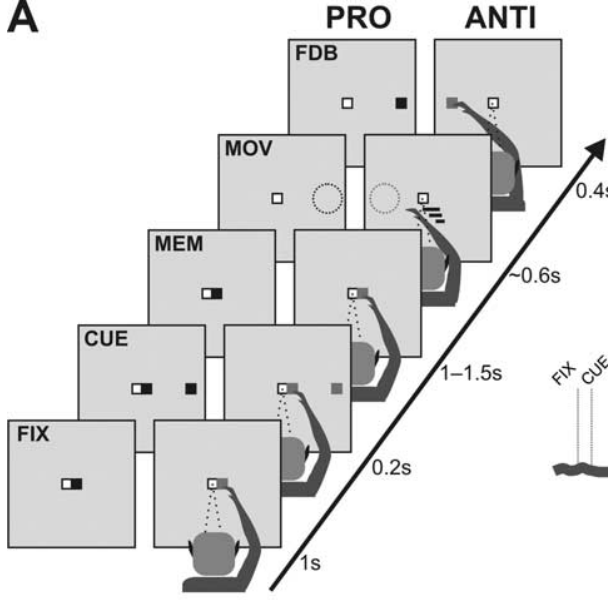

B

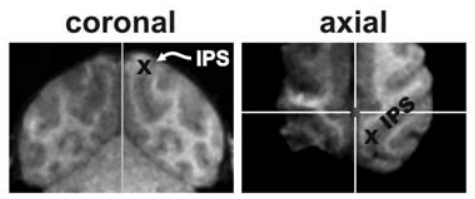

C

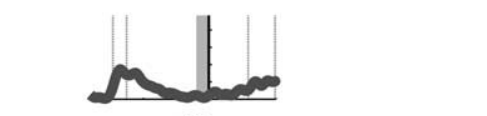

Figure 1. A, Memory-guided anti-reach task. The monkey had to keep ocular fixation on a small central red dot throughout the trial. The color of the central hand stimulus (green/blue) instructed randomly interleaved pro- or anti-reaches with the movement goal (dotted circles; not visible to the monkey) either at the location (left, right, up, or down) of the previously flashed cue (CUE) or opposite of it. Disappearance of the central hand stimulus after a variable memory period (MEM) instructed the movement (MOV). Visual feedback (FDB) appeared only after the monkey touched the correct goal on the screen (success) or after a wait time had expired short enough not to allow posture corrections (failure). $\boldsymbol{B}$, Localization of recording sites (cross indicates estimated center) in the PRR in the medial intraparietal sulcus (IPS) of monkey TI. (Shaded areas in the left one-half of the axial view are imaging artifacts.) C, Typical delay period tuning in PRR. Outer panels, Response profiles (PSTHs; aligned with movement instruction at $t=0$; smoothed with $50 \mathrm{~ms}$ Gaussian kernel) of a single example neuron strongly selective for the four different locations of the cue/reach goal during the standard memory-guided pro-reach. The inner panel shows the corresponding tuning curve with the resulting directional tuning vector (see Materials and Methods) for the late memory period (shaded area in PSTHs). Smoothed PSTHs or interpolated curves were only used for illustration; all quantitative analyses were performed based on direct spike counts.

Everling et al., 1999; Gottlieb and Goldberg, 1999; Everling and Munoz, 2000; Zhang and Barash, 2000) permitted the time-continuous assessment of sensory- versus motor-related spatial tuning of neural activity (Georgopoulos et al., 1989; Lurito et al., 1991; Zhang et al., 1997; Zhang and Barash, 2000, 2004). The dynamics of the sensoryto-motor transition revealed context-specific visuomotor transformations in PRR. During the delay period of a memory reach task, PRR exclusively represented motor goals, not visual memories. The latencies of these neural motor goal representations corresponded with subjects' reaction times, being higher for the incongruent spatial stimulus-response (S-R) mapping during anti-reaches.

\section{Materials and Methods}

Anti-reach task and control of behavioral parameters. Two male rhesus monkeys (Macaca mulatta) were trained to perform a visually instructed, center-out anti-reach task in a frontoparallel plane. Two versions of the task were used, a memory task (Fig. 1A) and a reaction time task. The memory task was used for neural recordings to analyze the PRR activity with respect to motor goal representations during the delay period (as opposed to motor representations during motor preparation/execution). The reaction time task was used to test for behavioral effects of the task rule (pro/anti) on the speed of sensorimotor transformations.

In the memory task, the monkey initiated a trial by fixating a small central, red fixation spot and touching an adjacent green or blue hand target (fixation period). The green touch target instructed a pro-reach trial, and the blue target an anti-reach trial. After a fixed delay $(1.0 \mathrm{~s})$, a peripheral visual cue of the same color as the central hand target was briefly flashed (cue period, $0.2 \mathrm{~s}$ ), indicating to the monkey where he later will have to reach. For a variable duration, the monkey had to keep the hand fixation (memory period, $1.0-1.5 \mathrm{~s}$ ). When the central hand target turned off (go signal), the monkey had to reach (movement period, maximum, $1.0 \mathrm{~s}$ ) either to the memorized location of the previously flashed cue (pro-reach; green cue) or to the diametrically opposed position (anti-reach; blue cue). The monkey received visual feedback about the correct movement goal (circular patch stimulus at the motor goal location) as soon as he acquired the desired position, or after the maximum movement period expired, in which case the trial was aborted. The monkey had to keep his hand at the reach target location (feedback period, $0.3 \mathrm{~s}$ ) to successfully finish the trial and receive liquid reward.

In the reaction time task, the monkeys had to reach toward the movement goal immediately after the visual cue was presented. The memory period was omitted in this case, and the maximum allowed movement period (reaction plus movement time) was reduced to $0.7-0.8 \mathrm{~s}$. In every other respect, the reaction time task was identical to the memory task described above. Reaction time was defined as the time between the movement instruction and the monkey's release of the touch screen. Movement time was defined as the time between the release and reacquisition of the touch screen at the target position.

Both versions of the anti-reach task consisted of eight different trial conditions (four target positions by two mapping rules, pro/anti). The four possible peripheral reach targets (right, $0^{\circ}$; up, $90^{\circ}$; left, $180^{\circ}$; down, $270^{\circ}$ direction) were pseudorandomly interleaved from trial to trial. Eccentricity was always $9 \mathrm{~cm}$ (corresponding to $17-20^{\circ}$ visual angles for screen distances of $25-30 \mathrm{~cm}$ for the two monkeys). Pro- and antireaches were also pseudorandomly interleaved; except for 12 of the 60 recording sessions total, when a block design was used ( 80 trials per block). The data from the block-wise recordings yield no differences to the randomized recordings and, therefore, will be presented jointly.

Eye position was registered with an infrared CCD camera $(240 \mathrm{~Hz}$; ISCAN, Burlington, MA). The monkeys had to keep ocular fixation throughout the course of the trial (tolerance, $3.5-4.0^{\circ}$ visual angle). Hand position was registered with a 19 inch touch screen (IntelliTouch; ELO Systems, Menlo Park, CA). The hand stimulus had to be continuously touched within a tolerance window of typically $1.8 \mathrm{~cm}\left(3.5-4.0^{\circ}\right.$ visual angle at $25-30 \mathrm{~cm}$ screen distance) for the central, and $2.5-3.5 \mathrm{~cm}$ $\left(4.8-8.0^{\circ}\right)$ for the peripheral targets. Otherwise, the trial was immediately aborted without reward. Visual instruction stimuli were either presented on a liquid crystal display monitor ( 19 inch ViewSonic VX924; $<5$ ms off-on-off response time) mounted behind the touch-sensitive screen, or rear-projected from a digital light processing projector (U2870; Plus Corporation, Tokyo, Japan) onto a tangent screen attached to back of the touch-sensitive screen. Visual display latencies were recorded with photo diodes for both systems and corrected for in the data analysis.

Animal preparation and recording procedure. Both monkeys first were implanted with a head holder. After the monkeys had learned the task, recording chambers were implanted above the posterior parietal cortex, contralaterally to the handedness of each monkey, to allow access to the medial wall of the intraparietal sulcus. Structural magnetic resonance imaging was used to identify the position of the intraparietal sulcus for placement of the recording chambers and guidance of the recordings (Fig. $1 B$ ). Surgical and imaging procedures were conducted under general anesthesia. All procedures were done in accordance with National Institutes of Health guidelines and were approved by the California Institute of Technology Animal Care and Use Committee.

Extracellular neural recordings were made from up to five microelectrodes simultaneously using a five-channel microdrive ("mini-matrix"; Thomas Recording, Giessen, Germany). The raw signal from each electrode was preamplified (20×; Thomas Recording), bandpassed, and amplified (154 Hz to $8.8 \mathrm{kHz}$; $400-800 \times$; Plexon, Dallas, TX), before being subjected to on-line spike-sorting (Sort Client; Plexon). All spike waveforms were digitized $(40 \mathrm{kHz})$ and recorded to disk for off-line control of 
sorting quality and stationarity (Offline Sorter; Plexon). In some sessions, a single-channel drive (Narishige, Tokyo, Japan) was used in combination with other electrodes (FHC, Bowdoinham, ME) and a $20 \times$ gain head stage (Plexon), but otherwise identical signal processing.

Single-cell spiking activity in the cortex beneath the recording chamber in each monkey was mapped for reach-related, delay-period activity to define a region of interest that conforms with the functional definition of PRR reported previously (Snyder et al., 1997). Recording sites were within 5-10 $\mathrm{mm}$ posterior and 4-7 mm lateral (Horsley-Clarke coordinates) at 3-6 $\mathrm{mm}$ subsurface, in the hemisphere contralateral to the arm used for reaching (monkey TI, left arm; TA, right). Data from this mapping procedure were not included in the current study. From independent subsequent recordings in this region, all units were included in the analysis that were sufficiently well isolated and stationary, regardless of their task-relatedness or specific tuning properties.

Neural tuning analysis. Single-cell spiking activity from successful memory reach trials were analyzed for spatial selectivity on a cell-by-cell basis (tuning analysis), and on the population level (see below, Neural decoding analysis).

In the tuning analysis, the selectivity of single-cell spike rates for the location of the visual cue/motor goal was assessed at different times relative to stimulus or behavioral events occurring during the trial. Fixed, predefined periods (each $200 \mathrm{~ms}$ ) were used for the tuning analysis: the last $200 \mathrm{~ms}$ before cue stimulus onset (baseline), the $200 \mathrm{~ms}$ of cue visibility (cue), the time between 100 and $300 \mathrm{~ms}$ after cue offset (early memory), the last $200 \mathrm{~ms}$ before the go signal (late memory), the last 200 $\mathrm{ms}$ before reach target acquisition (movement), and $200 \mathrm{~ms}$ after target acquisition during the visual feedback (feedback). Because the memory period and the arm movement itself had variable duration across trials, the neural signals were aligned either with respect to the cue onset, the end of memory period, or the time of the target acquisition to calculate averages. Spatial selectivity was quantified with a directional tuning vector (DTV), separately for pro- and anti-reaches. It is defined as vector average across all center-out cue directions $\overrightarrow{\mathrm{u}}_{i}$ (unit vectors) weighted with the corresponding mean spike rates $r_{i j}$ of neuron $j$ as follows:

$$
\operatorname{DTV}_{j}=\sum_{i=1}^{4} r_{i j} \vec{u}_{i} .
$$

The mean spike rate is calculated within each period and averaged across trials with identical conditions ( $\sim 15-20$ repetitions per condition). The direction of the resulting tuning vector defines the preferred direction of a neuron (for an example, see Fig. $1 C$ ). The DTV was calculated relative to the location of the cue (instead of the motor goal), which is an arbitrary choice that does not in any way affect the results. Significance of directional tuning was tested with a nonparametric one-way ANOVA (Kruskal-Wallis) with the four different visual cue directions as groups and sample sizes defined by the number of identical trial repetitions.

Four main tuning categories were defined based on the presence (or absence) of tuning in the pro- and the anti-reach condition. In each period, a neuron could either be tuned in both conditions (category I), in one of the two conditions [pro-only (II) or anti-only (III) tuning], or in none of the conditions (IV). For the pro and anti tuning category (I), three spatially defined subcategories were built based on a second factor, the relative preferred direction between pro- and anti-reaches. In case a neuron was tuned in the pro and anti condition, the difference of preferred directions between pro- and anti-reach tuning could be $<45^{\circ}$ (visual tuning), $>135^{\circ}$ (motor tuning), or in-between (spatially undefined tuning).

Neural decoding analysis. In the decoding analysis, we used onenearest-neighbor classification to quantify how informative the neural population response is about individual task parameters (Duda et al., 2001). We used classification procedures with different class definitions (class, set of trials with the task parameter in question kept constant) to predict different task parameters. With an eight-way classifier, trials were classified with respect to all possible task parameter combinations ("total decode," four cue directions by two task rules). With a two-way classifier, trials were classified only with respect to the task rule, regardless of cue/ motor goal direction ("context decode"). With a modified four-way classifier, trials were classified with respect to cue/motor goal direction. Trials with one task rule (pro-reaches) were used to train the classifier, whereas trials with the other task rule (anti-reaches) were used as test trials ("transconditional decode").

Similarity of the neural population response patterns between test and training trials is used to predict to which class a test trial belongs. In the one-nearest-neighbor algorithm, class membership of a test trial is predicted by the class membership of the training trial whose neural response vector has minimal Euclidian distance ("nearest neighbor") to the response vector of the test trial in the $\mathrm{N}$-dimensional space. $\mathrm{N}$ corresponds to the total number of neurons (see below). The neural response vector $\vec{r}_{i}(t)=\left(r_{1 j}(t), \ldots, r_{N j}(t)\right)$ is time dependent, where $r_{i j}(t)$ represents the spike rate of neuron $i$ in the time interval $t$ of trial $j$. The classification procedure was performed separately for each time interval $t$. Time intervals were $100 \mathrm{~ms}$ long with successive intervals overlapping by $50 \mathrm{~ms}$.

Decoding performance is the percentage of correctly predicted test trials. The classifier was trained with six of seven of all relevant trials (training set) and predictions were made for the remaining one of seven of the trials (test set) for all seven permutations of the sets (sevenfold cross-validation; see below for the total number of trials). Initial assignment of individual trials to the seven subsets was done at random. Decoding performances were averaged across the seven individual crossvalidation results. Deviation of decoding performances from chance level, or differences in performances between different classification conditions (e.g., decoding the motor goal in pro- vs anti-trials only), were tested with a $t$ test. The significance criterion was Bonferroni-corrected for the number of nonoverlapping time windows $\left(N_{t}=15 ; 30\right.$ time steps with $50 \%$ window overlap) to account for multiple testing induced by the time-dependent analysis.

The population of neurons was collected over multiple experimental sessions with a variable number of trials recorded for each neuron (typically $\sim 20$ trials per condition). For the classification analysis, the same number of trials $(=15)$ for each of the eight possible classes (four direction by two task rules) was selected as a random subsample of all trials recorded for this neuron. Only neurons were included in the decoding analysis for which a minimum of 15 trials per trial parameter combination was recorded ( $N=131$ of 143 recorded neurons). We did not preselect the neurons in any other way, which means the population decode includes all well isolated neurons, even if they are not significantly spatially tuned or task related based on the single-cell analysis.

Spatial selectivity of the neural activity only emerged after a spatial cue has been presented in each trial. To test for latency differences in the onset of spatial selectivity in pro- versus anti-reaches on the population level, we compared the dynamics of the decoding performances $P_{P}$ and $P_{A}$ between the two task conditions (pro/anti). For this, we calculated a cross-correlation function between the two performance curves (see Fig. $5 A$ ) as a function of time. The relative time shift $\Delta t$ between the two temporal performance profiles was calculated as the time lag of the peak in the unbiased cross-correlation function:

$$
C_{P A}(\mathrm{~m})=\left\{\begin{array}{cc}
\frac{1}{N-|m|}\left(\sum_{n=0}^{N-m-1} P_{p}(n+m) P_{A}(n)\right) m \geq 0 \\
C_{P A}(-m) & \text { else }
\end{array}\right.
$$

Arguments $n$ and $m$ denote indices for the time bins. To increase the temporal resolution for the calculation of the time shift, the performance profiles $P_{P}$ and $P_{A}$ were interpolated by a factor of 25 before crosscorrelating ("ideal" low-passing via fast Fourier-transform; MATLAB function interp), leading to a nominal $2 \mathrm{~ms}$ resolution. The time lag measure is sensitive to small differences in the exact shape of the two performance curves. This shape can vary slightly depending on the random trial selection in the cross-validation procedure. To estimate the reliability of the time lag measure, we averaged across 100 repetitions of calculating the performance curves with different random trial selections.

Other classification algorithms, such as maximum likelihood or Fisher's linear discriminant analysis, were tested also, yielding qualitatively 

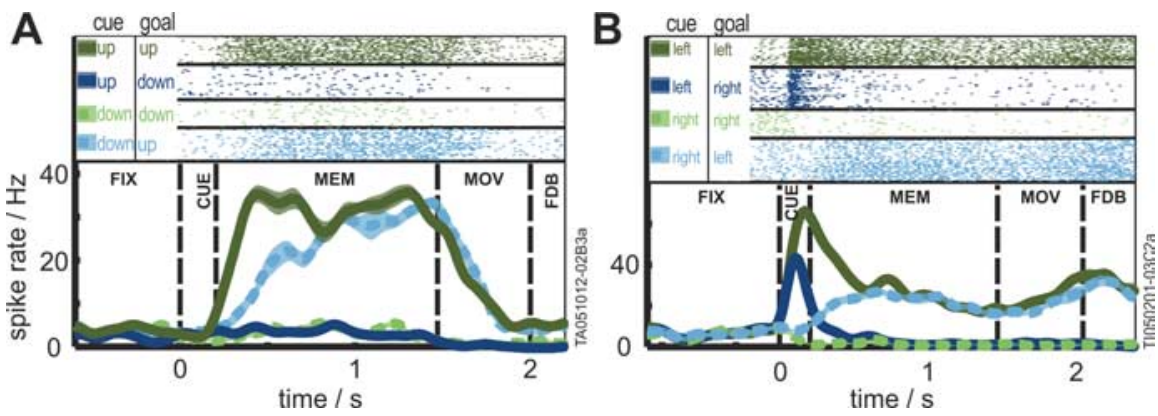

Figure 2. A, Example neuron with sustained motor goal tuning. A comparison of the PSTHs for up (dark, continuous) and down (light, dotted) cue positions during pro- (green) and anti-reaches (blue) shows that this neuron is only activated by a motor goal "up," independent of the cue position. Note that, for each neuron, all eight possible conditions were recorded; responses for the remaining four conditions were intermediate and are not shown for convenience. $\boldsymbol{B}$, Example neuron with visuomotor tuning. Conventions are as in $\boldsymbol{A}$ only with left/right instead of up/down positions shown. This neuron is first tuned for left-side cues, and later for left-side motor goals. Abbreviations are as defined in the legend to Figure 1.

the same results. Because the general conclusions are not affected by the choice of the classification algorithm, we decided on the nearestneighbor algorithm because of its simplicity, robustness, and lack of assumptions on the shape of spike rate distribution or directional tuning functions.

\section{Results}

Visual versus motor goal tuning

Extracellular single-cell spiking activity was recorded from 143 PRR neurons (90 monkey TI; 53 monkey TA) while the monkeys performed the memory anti-reach task. Results in both monkeys were qualitatively the same and will be presented jointly.

By comparing the directional tuning of the neurons in the pro- versus anti-reach conditions, we were able to discriminate neural representations of visual sensory information (cue location) from motor goal information (reach target location) over the course of the trial. Figure 2 shows the peristimulus time histograms (PSTHs) of two example neurons for pro-/anti-reaches in the preferred and the opposite direction. Both neurons show different time courses of their neural responses. Neuron A is exclusively active during the memory period in two of the four task conditions shown. The two active conditions shared a common motor goal, in this case an upward $\left(90^{\circ}\right)$ reach target, but with opposite-side visual instruction cues. In the two downward $\left(270^{\circ}\right)$ reach conditions, the neuron was inactive, independent of the position of the cue. This means that example neuron A represented the location of the monkey's motor goal during the entire memory period. At no time did the activity of this neuron contain information on the position of the visual cue. We will call this type of spatiotemporal selectivity "motor goal tuning" ("goal" because the tuning appears long before the actual reach movement itself); it is defined by significant spatial tuning during the memory period in both, the pro- and anti-reach trials, with the preferred directions in the two conditions being opposite to each other when calculated with respect to the cue direction (i.e., being identical when calculated with respect to the reach direction). Neuron B, in contrast, had a spatiotemporal "visuomotor tuning." This is characterized by a selectivity of a neuron for the spatial location of the instructive cue during the presence of the cue, changing into selectivity for the spatial location of the motor goal some time during the memory period. At any time the neuron was active during two of the four conditions shown. Immediately after cue onset, the neuron had high firing rate for leftward $\left(180^{\circ}\right)$ cues, regardless of the reach goal (spatially visual tuning). In contrast, from early on in the memory period, the neuron was highly active only in case of leftward reaches, regardless of the previous cue location (spatially motor goal tuning).

In the following analyses, we will quantify the relative contributions of visual versus motor goal tuning to the overall neural representation in PRR. We used decoding analysis for time-resolved analysis of the population response, and tuning analysis in predefined time windows to track the contributions of the individual neurons to the population behavior. We will focus on the transition from the cue period to the memory period; later periods are dominated by spatial tuning for the direction of the motor goal and will not be the focus of this study.

Decoding analyses were used to predict different task parameters based on the population response of all recorded neurons (131 of 143 with sufficient number of trials for this analysis; see Materials and Methods). In the complete decode, we classified the total set of different trial conditions (four directions by two rules). The decoding performance in this case is a measure of how much of the combined information on the cue/motor goal direction and the mapping rule (the behavioral context) is contained in the population activity. One hundred milliseconds after cue onset, total prediction performance rose steeply above chance level (12.5\%), peaking at $\sim 90 \% \sim 150 \mathrm{~ms}$ after the start of the memory period (50 ms resolution) (Fig. $3 A$ ). The prediction performance increased monotonically with the number of neurons (Fig. $3 A$, inset).

The prevalence of sensory or motor-related spatial tuning cannot be derived from the total decode performance. To achieve this dissociation, we performed a transconditional decode. The classifier was trained on the four directions in pro-reach trials only (cue and reach-goal directions are identical in this case) and used to predict a direction in anti-reach trials. This predicted direction was compared with the true direction of either the cue or the reach-goal direction in the anti-reach trials (Fig. 3B). The idea behind the transconditional decode is that if PRR at any given time mainly represents visual cue information, then the classifier trained on pro-trials should be able to predict the cue direction in anti-trials. If PRR mainly represents motor goal information, then the classifier trained on pro-trials should be able to predict the reach direction in anti-trials. PRR population response shows a moderate $(<60 \%)$ prediction performance for the cue direction at $\sim 100 \mathrm{~ms}$ latency relative to cue onset. This visual cue representation vanishes quickly and, already by the end of the cue period, is replaced by a motor goal representation, indicated by a significant prediction performance for the reach direction. This motor goal prediction increases to $>90 \%$ during the memory period. Note, because cue and reach directions are opposite in anti-reaches, correct predictions of visual cue and motor goal are mutual exclusive, when the training is based on pro-trials, where the two directions are identical. This explains the anti-correlated performance curves in Figure $3 B$, "mirrored" at the chance level (25\%). Note also that the prediction performance of the transconditional classification analysis per se does not imply that there is no visual cue information encoded in the population activity during the memory period. It only indicates a strong predominance of motor goal tuning over visual tuning. The additional analysis of the tuning of individual neurons, how- 

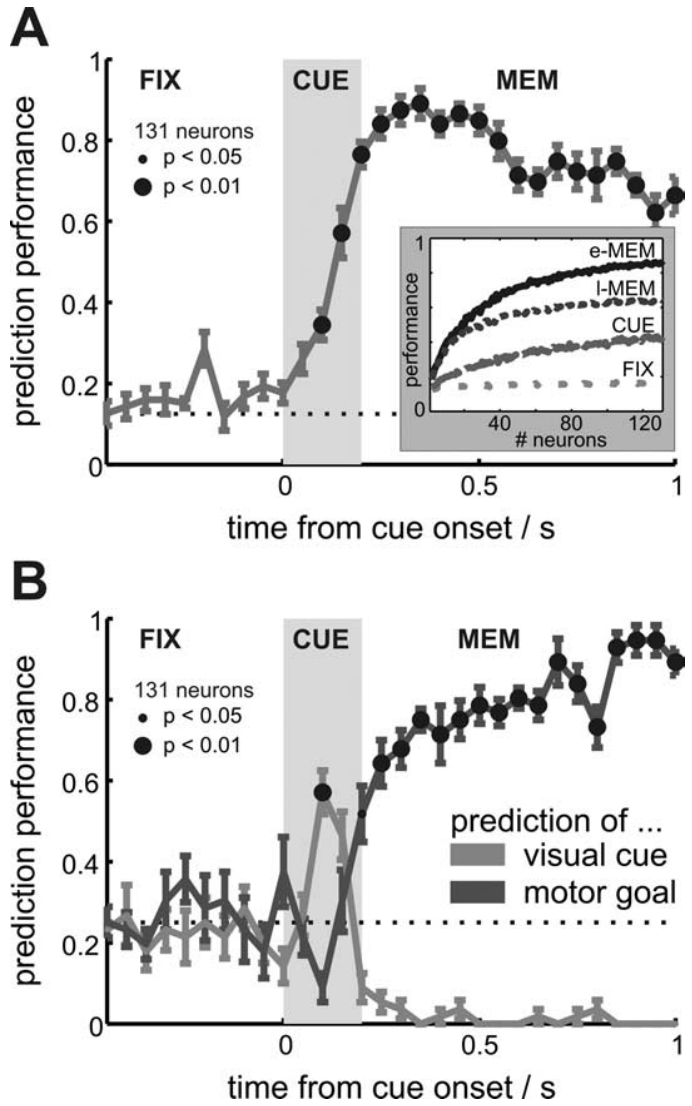

Figure 3. A, Time course of total decode performance and learning curves. The performance for predicting all eight trial conditions ( 4 directions by 2 task rules; $12.5 \%$ chance level) becomes significant $\sim 100 \mathrm{~ms}$ after cue onset and reaches its maximum during the early memory period (e-MEM). The decline in the later memory period (I-MEM) can be explained by the prevalence of pure motor goal tuning of most neurons at this time, which allows decoding the motor goal well (Fig. 3B), but the task rule poorly (Fig. 6). Inset, Prediction performance improves monotonically with the number of neurons used for classification. Note that the neurons were randomly selected among all neurons recorded in the region of interest, regardless of their tuning properties or task relatedness (see Materials and Methods). B, Dynamics of sensory versus motor representations. The classifier was trained with pro-reach trials only (4-way classification of direction; $25 \%$ chance level), and then used to predict a direction in anti-reach trials. The performance value denotes the probability that the predicted direction coincides with the cue or motor goal position, respectively. This decode revealed a moderate transient representation of the cue position during part of the cue period (light curve). This sensory representation is then quickly replaced by a strong motor goal representation in the population of PRR neurons (dark curve). Note that correct prediction of the motor goal and the cue are mutually exclusive in this kind of decode, which explains the below-chance performances (see Results). Because of this statistical dependence, performance values for the cue versus motor goal prediction were each tested for being larger than chance level ( $t$ test, $p<0.05 / 0.01$ ), not for being different from each other. Other abbreviations are as defined in the legend to Figure 1. Error bars indicate SEM.

ever, showed that indeed there are no neurons with sustained visual tuning (see below).

How do individual neurons contribute to the transformation of a cue into a motor goal representation? It could be that this transformation is seen individually in the response profile of each neuron, as in example neuron B with visuomotor tuning (Fig. 2). Alternatively, different subpopulations of neurons could encode either the visual cue or the motor goal alone; where the first group shows transient activation during the cue period, and is complemented by a second group that "takes over" at the beginning of the memory period. Neuron A with motor goal tuning during the memory period represents an example of the second group (Fig. 2). To quantify the different spatiotemporal tuning profiles, first, we characterized individual neurons by assigning them to a cer-

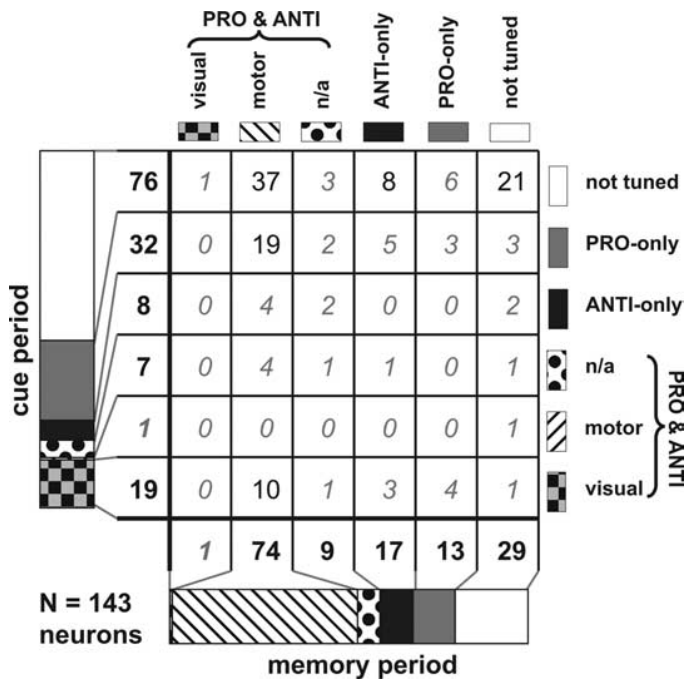

Figure 4. Spatiotemporal tuning classes. Comparing spatial tuning of all 141 neurons in cue and late memory period reveals few major classes (classes with population densities $<5 \%$ are in italics): (1) neurons with pure motor goal tuning [not tuned (37) or tuned during pro-reaches only (19) in the cue period, and tuned for the motor goal during the memory period], (2) neurons with visuomotor tuning [visual tuning during cue period, motor goal tuning during memory period (10)], (3) very few neurons specifically tuned during pro- or anti-reaches only [mostly during the memory period ( 6 of 8 )], and (4) neurons not tuned in both cue and memory period (21). Neurons that are tuned neither during the cue nor the memory period might be tuned during later periods of the trial. We here focused on the transition from the cue to the memory period and discuss the motor goal and sensorimotor neurons, because we were mainly interested in the neural dynamics of the cue-to-goal representation in PRR.

tain spatial tuning category and, second, compared the spatial tuning category of a neuron between the cue and the late memory period. We chose the late instead of the early memory period to capture also neurons that develop their tuning not immediately but later during the memory period (see below). The spatial tuning category was determined by two factors, the presence (or absence) of tuning in the pro- and the anti-reach condition, and the relative preferred direction between pro- and anti-reach tuning in the case in which a neuron was tuned in both conditions (for details, see Materials and Methods). The persistency rates for staying within a spatial tuning category, and the conversion rates from one to another spatial tuning category, respectively, during the transition from the cue to the memory period are shown in Figure 4. Several observations can be made: First, only few neurons in the cue period were visually tuned ( 19 of $143 ; 13 \%$ ), most of which later became motor-tuned (10 of 19; 53\%). Second, there were practically no neurons that were tuned exclusively during the cue period or had sustained visual tuning. Conversion rates from any kind of tuning during the cue period to not being tuned during the memory period were all $<3 \%$, and hence considered coincidental. Third, most neurons showed motor goal tuning in the memory period (74 of $143 ; 52 \%$ ), many of which have not been tuned during the cue period ( 37 of $74 ; 50 \%$ ), and if so, then often only during pro-trials ( 19 of $74 ; 26 \%$ ). In summary, there were only few neurons (10 of $143 ; 7 \%$ ) with visuomotor tuning as introduced with example neuron B (Fig. 2), whereas there were many neurons that show motor goal tuning as soon as they are consistently tuned in both the pro- and anti-reach conditions, without ever having been visually tuned [(74 - 10)/ $143=45 \%$ ], as is the case in example neuron A (Fig. 2).

Corresponding analysis for the early memory period revealed the same trend as found in the late memory period. The prevalence of motor tuning was less pronounced in the early memory 
period, as can be expected from the increasingly strong motor goal representation seen in the time-continuous decoding analysis (Fig. 3B).

\section{Latency of motor goal tuning}

The spatial tuning emerges faster in the pro- compared with the anti-reach trials. This can already be seen from example neuron A (Fig. 2), in which the neural activation for the preferred direction in pro-reaches has shorter latency than for anti-reaches to the same direction. The fact that during the cue period many neurons are spatially tuned exclusively during the pro-trials (32 of 143; $22 \%$ ), whereas this is hardly the case for anti-trials ( 8 of $143 ; 6 \%$ ), further indicates that the shorter tuning latency in pro-trials denotes a general trend (Fig. 4). Note that most of the neurons that initially are only tuned during the pro-trials later are tuned in both task conditions, and then represent the motor goal ( 19 of 32; $59 \%$ ). This makes the assumption plausible that the early tuning in the pro-trials already represents motor goal tuning, although a strict definition of motor goal tuning would require the comparison of spatial tuning between pro- and anti-reaches, which is not possible in this case. To quantify the difference in tuning latency between the pro- and anti-reach conditions, we evaluated the prediction performance separately for the pro- and anti-reach trials. This was done by calculating the performance for all pro-/ anti-trials as a subset of all trials contributing to the total decode (eight-way classification). The prediction performance as a function of time is delayed by $56 \pm 8 \mathrm{~ms}$ (mean $\pm \mathrm{SD} ; t$ test, $p<0.01$ ) in anti- compared with pro-reach trials (Fig. $5 \mathrm{~A}$ ) (for the calculation of the delay, see Materials and Methods), indicating that overall spatial information is represented earlier in pro- compared with anti-reach conditions.

\section{Reaction time task}

A delayed spatial tuning in anti-reaches is plausible when assuming that anti-reaches require an additional geometrical computation of the reach goal ("inversion vector"), in contrast to proreaches, and that this computation takes time. To behaviorally test for the existence of such an additional time-consuming process in anti- compared with pro-reaches, we let the monkeys perform both types or reaches in a reaction time task without instructed delay (see Materials and Methods). An instructed delay would obscure any reaction time difference related to the computation of the reach goal because the memory period lasts long enough for the monkey to decide on either reach goal, congruent (pro-reaches) or incongruent (anti-). Therefore, in the memory task, no reaction time differences were expected, which was the case in both monkeys (data not shown). In contrast, in the reaction time task correct pro-reaches are performed significantly faster than correct anti-reaches by 38-50 ms (confidence interval for difference in mean reaction times, $\alpha=0.01$ ) (Fig. $5 B$ ).

In our reaction time task, we found no behavioral indications for early involuntary pro-reaches during anti-reach trials, as might be expected from respective anti-saccade findings. The likelihood of miss-reaches in anti-trials was not higher in fast $(\mathrm{RT}<$ median $\mathrm{RT})$ compared with slow $(\mathrm{RT}>$ median $\mathrm{RT})$ trials $\left(\chi^{2}\right.$ test; monkey TA, $p>0.05, N=1728$; TI, $p=0.046, N=$ 2208). Also, the likelihood in anti-trials of miss-reaches to the pro-reach target, compared with other distracter targets, was not higher in fast compared with slow trials ( $p>0.05$, each monkey).

\section{Task-rule-dependent activity}

The fact that the tuning of PRR neurons depends on the behavioral context (pro vs anti mapping rule) suggest that PRR activity
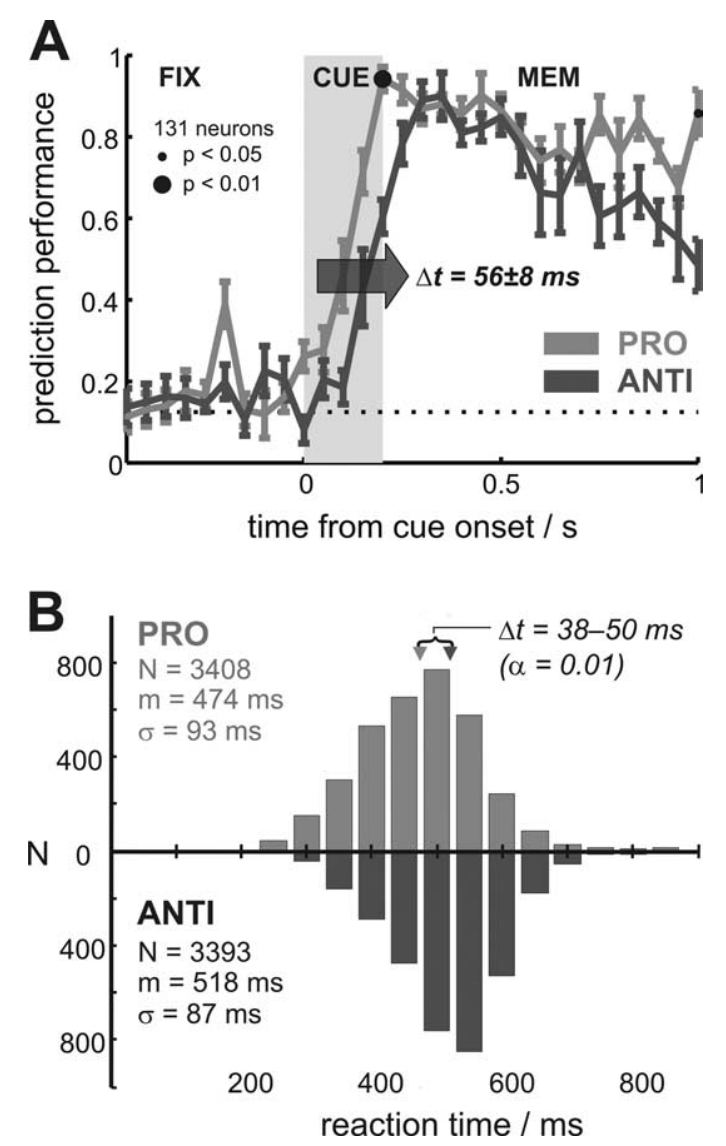

Figure 5. $\quad \boldsymbol{A}$, Motor goal representation in pro- versus anti-reaches. Prediction performance for the motor goal was delayed in anti- (dark) compared with pro-reaches (light). Curves show the average performance in the eight-way total decode ( $12.5 \%$ chance level) when analyzed separately for pro- and anti-reach trials. The delay (56 $\pm 8 \mathrm{~ms}$ ) was calculated by crosscorrelating the two performance curves (see Materials and Methods). $\boldsymbol{B}$, Reaction times in proversus anti-reaches. Arm movement onset of correct trials in a speeded reaction time task was delayed in anti- compared with pro-reaches. [Data were collected at 6 (monkey TA) and 4 (TI) separate days in the highly trained animals.] Abbreviations are as defined in the legend to Figure 1. Error bars indicate SEM.

contains at least implicit contextual information. By "implicit," we mean information that allows inferring on the currently valid behavioral context from the population activity of the neurons. But this information could be conveyed indirectly by the fact that the relative tuning properties of the neurons are different in the two conditions. Explicit contextual information, in contrast, is present when the behavioral context can be inferred independently from the spatial tuning of the neurons. Figure 6 shows that a two-way classification of the behavioral context becomes significant already during the fixation period (i.e., before any spatial information on the cue location/motor goal was available to the monkey, and hence before any spatial tuning in the neural activity occurred). This strongly indicates the existence of explicit contextual information in PRR activity in addition to, and independent from, the spatial tuning. Whereas normalized spike rates across the population of recorded neurons did not on average show a significant difference between pro- and anti-reaches in the fixation period $200 \mathrm{~ms}$ before cue onset $(p>0.05$, Wilcoxon signed-rank test), significant spike rate differences between proand anti-reaches in this period could be found in 21 individual neurons. Sixteen neurons showed stronger activation during pro-reaches, and five neurons during anti-reaches. Spike rates 


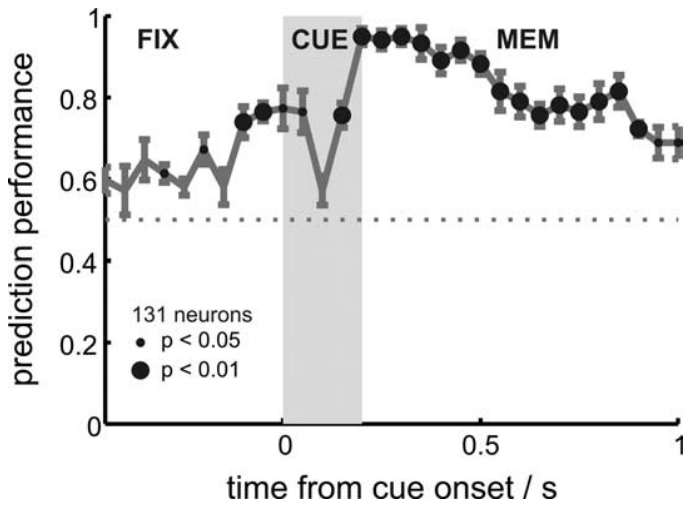

Figure 6. Representation of the task rule in PRR. The prediction of the task rule (mapping conditions, pro/anti) was significantly above chance (50\%) already shortly before the cue period (note that the fixation period had constant duration), indicating explicit task rule representations in PRR. Prediction was best at the end of the cue period and during the early memory period. This is probably attributable to additional implicit task rule information conveyed by the different relative spatial tuning of the neurons in pro- and anti-trials (see Discussion). Abbreviations are as defined in the legend to Figure 1. Error bars indicate SEM.

during the fixation (and other) periods were highly variable across neurons. Neurons with context modulation during the fixation period tended to have higher spike rates than nonmodulated neurons in this period ( $p<0.05$, rank sum test). Spike rates (averaged across all trials) ranged from 0.9 to $46 \mathrm{~Hz}$ with a median of $6.6 \mathrm{~Hz}(25 / 75 \%$ quantiles, $3.3 / 15 \mathrm{~Hz})$ for the 21 contextmodulated neurons, and from 0 to $34 \mathrm{~Hz}$ with a median of $3.8 \mathrm{~Hz}$ $(25 / 75 \%$ quantiles, $1.5 / 7.9 \mathrm{~Hz})$ for the population of nonmodulated neurons.

\section{Discussion}

The parietal reach region immediately translates current sensory information into reach movement plans (i.e., it represents spatial information about the goal of a pending movement rather than the previous cue during the delay period of a memory task). The integration of spatial sensory information with the task rule (pro/ anti) takes longer for incongruent compared with congruent visuomotor mappings. The latency difference in the occurrence of the motor goal representation is consistent with longer reaction times for anti-reaches compared with pro-reaches.

\section{Context-specific mapping: sensory versus motor-related activity}

In previous delayed-response experiments, the temporal proximity of neural responses to the sensory or behavioral motor event, respectively, was used as a plausibility argument for the sensor- or motor-relatedness of neural activity (Fuster, 1973; Hikosaka and Wurtz, 1983; Andersen et al., 1987; Gnadt and Andersen, 1988). The proximity argument does not work for sustained activity in the delay period between sensory cue and motor response. This led to contradictory interpretations of sustained PPC activity either representing sensory memory/spatial attention (Colby et al., 1996; Gottlieb et al., 1998; Powell and Goldberg, 2000; Bisley and Goldberg, 2003) or motor planning/intention-related processes (Gnadt and Andersen, 1988; Bracewell et al., 1996; Mazzoni et al., 1996; Snyder et al., 1997).

With the anti-reach task, we could overcome this ambiguity by assessing time-resolved spatial selectivity for either the cue or the motor goal. We found moderate $(<60 \%)$ transient representation of the cue location during the cue period and the strong $(>90 \%)$ representation of the motor goal during the memory period (Fig. 3). This means, first, that the sustained "memory" activity cannot be explained by retrospective processes like shortterm visual memory of the cue. Second, the dynamic spatial tuning of the few (7\%) sensorimotor-type neurons (Fig. 2B) could represent either a transition from a visual to a motor goal representation, both in retinal coordinates, or a spatial shift of attention from the cue to the motor goal location, two alternatives that might denote different sides of the same coin, namely "selectionfor-action" (Allport, 1987; Rizzolatti et al., 1987). Because the time of the go signal was unpredictable and was the only behaviorally relevant stimulus event after the cue presentation, one would expect the monkey's spatial attention in this trial phase to shift at least in part toward the center of the screen. However, the motor goal tuning in the visuomotor-tuned neurons persists unchanged throughout the delay period (Fig. $2 B$ ) and, on the population level, is even strongest toward the end of the delay period (Fig. 3B). This makes the attention interpretation implausible even for the few visuomotor-tuned neurons (hence our choice of calling them "visuomotor"). Third, the spatial encoding of the many (45\%) neurons with sustained motor goal tuning (Fig. 2A) completely defies an interpretation in favor of attentional shifting, because these neurons exclusively represent the motor goal. The early onset of their motor tuning, long before the actual reach movement instruction, suggests that this activity is related to motor-intention or -goal representations, rather than motorpreparatory or -control activity.

The conclusion that sustained PRR activity during the delay period of a memory task represents mainly motor-related information is further supported by the fact that most PRR neurons are selectively active during planned reaches but not during planned saccades (Snyder et al., 1997). Anti-saccade experiments in the inferior parietal lobe (LIP) yielded mixed results with respect to the sensory versus motor dissociation, with one report indicating largely sensory tuning (Gottlieb and Goldberg, 1999) and another set of studies reporting prevalence of motor goal tuning similar to our PRR results (Zhang and Barash, 2000, 2004).

\section{Task rule representations in PRR}

The discriminability of pro- and anti-reaches during the fixation period (Fig. 6) indicates task rule-specific modulation of PRR activity. Because we used a color instruction to indicate the task rule to the monkey, the observed differences could be a modulation attributable to stimulus color. We consider this possibility very unlikely. First, the task rule can be decoded significantly above chance level only shortly before cue onset, whereas the colored instruction stimulus is present during the whole fixation period. Second, to our knowledge, there have been no reports of genuine color selectivity in PPC. Selective responses to colored instruction stimuli in PPC could always be attributed to either the stimulus-associated movement response (Snyder et al., 1997) or the associated task rule (Toth and Assad, 2002; Stoet and Snyder, 2004). The representation of arbitrary S-R associations is mostly associated with areas in the frontal lobe. Although the association between color and task rule in our case is also "arbitrary" in this sense, the content of the two task rules denote two different kinds of spatial S-R mappings, i.e., two different modes of visual guidance of the reach movement (toward or away from the cue location). Because PRR is known to be involved in visually guided reaching, the spatial nature of the task rule might explain the observed rule-specific modulations in PRR. More generally, because of its spatial competence, PPC might be recruited for the integration of abstract task rules with sensorimotor processing whenever spatial transformation rules are in effect (Toni et al., 2001). 


\section{Dynamics of context-specific sensorimotor transformations}

At least three separate processes contribute to a sensory-to-motor (goal) transformation in an anti-reach task: context categorization (pro/anti), cue identification, and spatial cue-to-goal mapping. The time to perform the cue-to-goal mapping depends on the S-R congruency and is longer for incongruent mappings by $\sim 50 \mathrm{~ms}$, corresponding to latency differences in the motor goal representations of PRR (Fig. 5). We argue that PRR reflects the computational costs of performing an incongruent cue-togoal mapping by a belated occurrence of its motor goal representations. We suggest that the response profile of the visuomotortuned "transformation" neurons might directly reflect the spatial cue-to-goal mapping procedure ("vector inversion”). Neurons with visuomotor tuning reverse their tuning dynamically during anti-reaches (e.g., they shut down after an initial activation, when the cue but not the motor goal was in their response field, and they become active after initial silence in the opposite case) (Fig. 2 B, blue curves). The time cost for changing the activation status of these transformation neurons during antireaches might explain the higher latency for reaches with incongruent spatial mapping. Motor-tuned neurons in PRR became spatially tuned after the switch in the transformation neurons had occurred. They represented the outcome of the spatial transformation once it was accomplished (the "verdict" of PRR) and probably denote the output stage of this area.

The cue-to-goal mapping took at most $100 \mathrm{~ms}$ in our data: It could not yet have been accomplished when PRR first established cue representations $(\sim 50-100 \mathrm{~ms})$ but must have been when the sensory-to-motor goal transformation was completed (150-200 $\mathrm{ms})$. The total time for a sensory-to-motor transformation in an anti-saccade experiment in LIP (Zhang and Barash, 2000) was much longer (average, $415 \mathrm{~ms}$ after cue offset) on the population level [for a similar time course, see also Bisley and Goldberg (2003)]. The difference might in part be explained by a cost for context categorization: Zhang and Barash (2000) provided the context information (pro/anti) with the spatial cue, which means context categorization could take place only during or after cue identification. We provided context information from the beginning of the trials. This allowed context categorization before cue identification, which is supported by our finding of rule-dependent neural activity during the fixation period (Fig. 6). Because individual "paradoxical" neurons in the Zhang and Barash (2000) experiment showed early context-specific, nonvisual responses $\sim 50-100 \mathrm{~ms}$ after response onset in LIP $(\sim 60$ $\mathrm{ms}$ ), it was suggested that context categorization does not exceed the latency for availability of spatial cue information to PPC areas by more than 50-100 ms (Barash, 2003). The remaining $>200$ $\mathrm{ms}$ for cue-to-goal mapping are slower than the estimated $\sim 100$ $\mathrm{ms}$ in our data. This could be a consequence of a need for "default-response cancellation" (Kornblum et al., 1990), which might be specific to anti-saccades. To successfully initiate an antisaccade, "reflexive" pro-saccades, as manifest in express saccades (Fischer and Boch, 1983; Fischer and Weber, 1993), have to be suppressed. From our reaction time experiment, we have no indication for an urge of the monkeys to perform pro-reaches in the early period of anti-trials. Should a corresponding covert "default" movement plan for pro-reaches nevertheless exist in antireach trials, it is noteworthy that the majority of neurons in PRR, which have sustained motor goal tuning, do not represent this "erroneous" reach plan.

\section{Conclusions}

Our experiment shows that PRR represents integrated knowledge on spatial sensory information combined with abstract behavioral rules (pro/anti) to represent the desired movement goal independent of the instruction stimulus as soon as all relevant information is available. The dynamic contribution of individual neurons to both visual and motor goal representations at different points in time underlines the notion that within the frontoparietal network the distinction between "sensory" and "motor" relatedness is often less an anatomical-physiological distinction, a label that could be assigned to individual areas or neurons based on its static tuning properties, but rather denotes a temporal aspect of the ongoing sensorimotor dynamics, shifting between sensory and motor tuning depending on the currently most relevant or available control signals.

\section{References}

Allport DA (1987) Selection for action: some behavioral and neurophysiological considerations of attention and action. In: Perspectives on perception and action (Heuer H, Sanders AF, eds), pp 395-419. Hillsdale, NJ: Erlbaum.

Andersen RA, Buneo CA (2002) Intentional maps in posterior parietal cortex. Annu Rev Neurosci 25:189-220.

Andersen RA, Essick GK, Siegel RM (1987) Neurons of area 7 activated by both visual-stimuli and oculomotor behavior. Exp Brain Res 67:316-322.

Barash S (2003) Paradoxical activities: insight into the relationship of parietal and prefrontal cortices. Trends Neurosci 26:582-589.

Batista AP, Buneo CA, Snyder LH, Andersen RA (1999) Reach plans in eyecentered coordinates. Science 285:257-260.

Battaglia-Mayer A, Ferraina S, Mitsuda T, Marconi B, Genovesio A, Onorati P, Lacquaniti F, Caminiti R (2000) Early coding of reaching in the parietooccipital cortex. J Neurophysiol 83:2374-2391.

Battaglia-Mayer A, Caminiti R, Lacquaniti F, Zago M (2003) Multiple levels of representation of reaching in the parieto-frontal network. Cereb Cortex 13:1009-1022.

Bisley JW, Goldberg ME (2003) Neuronal activity in the lateral intraparietal area and spatial attention. Science 299:81-86.

Boettiger CA, D’Esposito M (2005) Frontal networks for learning and executing arbitrary stimulus-response associations. J Neurosci 25:2723-2732.

Boussaoud D, Wise SP (1993a) Primate frontal cortex-neuronal-activity following attentional versus intentional cues. Exp Brain Res 95:15-27.

Boussaoud D, Wise SP (1993b) Primate frontal cortex-effects of stimulus and movement. Exp Brain Res 95:28-40.

Bracewell RM, Mazzoni P, Barash S, Andersen RA (1996) Motor intention activity in the macaque's lateral intraparietal area. II. Changes of motor plan. J Neurophysiol 76:1457-1464.

Buneo CA, Andersen RA (2006) The posterior parietal cortex: sensorimotor interface for the planning and online control of visually guided movements. Neuropsychologia, in press.

Buneo CA, Jarvis MR, Batista AP, Andersen RA (2002) Direct visuomotor transformations for reaching. Nature 416:632-636.

Burnod Y, Baraduc P, Battaglia-Mayer A, Guigon E, Koechlin E, Ferraina S, Lacquaniti F, Caminiti R (1999) Parieto-frontal coding of reaching: an integrated framework. Exp Brain Res 129:325-346.

Colby CL, Duhamel JR, Goldberg ME (1996) Visual, presaccadic, and cognitive activation of single neurons in monkey lateral intraparietal area. J Neurophysiol 76:2841-2852.

Crammond DJ, Kalaska JF (1994) Modulation of preparatory neuronal activity in dorsal premotor cortex due to stimulus-response compatibility. J Neurophysiol 71:1281-1284.

di Pellegrino G, Wise SP (1993) Visuospatial versus visuomotor activity in the premotor and prefrontal cortex of a primate. J Neurosci 13:1227-1243.

Duda RO, Hart PE, Stork DG (2001) Pattern classification. New York: Wiley.

Everling S, Munoz DP (2000) Neuronal correlates for preparatory set associated with pro-saccades and anti-saccades in the primate frontal eye field. J Neurosci 20:387-400.

Everling S, Dorris MC, Klein RM, Munoz DP (1999) Role of primate supe- 
rior colliculus in preparation and execution of anti-saccades and prosaccades. J Neurosci 19:2740-2754.

Fischer B, Boch R (1983) Saccadic eye movements after extremely short reaction times in the monkey. Brain Res 260:21-26.

Fischer B, Weber H (1993) Express saccades and visual attention. Behav Brain Sci 16:553-567.

Funahashi S, Chafee MV, Goldman-Rakic PS (1993) Prefrontal neuronal activity in rhesus monkeys performing a delayed anti-saccade task. Nature 365:753-756.

Fuster JM (1973) Unit activity in prefrontal cortex during delayed-response performance: neuronal correlates of transient memory. J Neurophysiol 36:61-78.

Georgopoulos AP, Lurito JT, Petrides M, Schwartz AB, Massey JT (1989) Mental rotation of the neuronal population vector. Science 243:234-236.

Gnadt JW, Andersen RA (1988) Memory related motor planning activity in posterior parietal cortex of macaque. Exp Brain Res 70:216-220.

Gottlieb J, Goldberg ME (1999) Activity of neurons in the lateral intraparietal area of the monkey during an antisaccade task. Nat Neurosci 2:906-912.

Gottlieb JP, Kusunoki M, Goldberg ME (1998) The representation of visual salience in monkey parietal cortex. Nature 391:481-484.

Grol MJ, de Lange FP, Verstraten FAJ, Passingham RE, Toni I (2006) Cerebral changes during performance of overlearned arbitrary visuomotor associations. J Neurosci 26:117-125.

Hikosaka O, Wurtz RH (1983) Visual and oculomotor functions of monkey substantia nigra pars reticulata. 3. Memory-contingent visual and saccade responses. J Neurophysiol 49:1268-1284.

Johnson PB, Ferraina S, Bianchi L, Caminiti R (1996) Cortical networks for visual reaching: physiological and anatomical organization of frontal and parietal lobe arm regions. Cereb Cortex 6:102-119.

Kalaska JF (1996) Parietal cortex area 5 and visuomotor behavior. Can J Physiol Pharmacol 74:483-498.

Kornblum S, Hasbroucq T, Osman A (1990) Dimensional overlap: cognitive basis for stimulus-response compatibility—a model and taxonomy. Psychol Rev 97:253-270.

Lurito JT, Georgakopoulos T, Georgopoulos AP (1991) Cognitive spatialmotor processes. 7. The making of movements at an angle from a stimulus direction-studies of motor cortical activity at the single-cell and population levels. Exp Brain Res 87:562-580.

Marconi B, Genovesio A, Battaglia-Mayer A, Ferraina S, Squatrito S, Molinari M, Lacquaniti F, Caminiti R (2001) Eye-hand coordination during reaching. I. Anatomical relationships between parietal and frontal cortex. Cereb Cortex 11:513-527.

Mazzoni P, Bracewell RM, Barash S, Andersen RA (1996) Motor intention activity in the macaque's lateral intraparietal area. I. Dissociation of motor plan from sensory memory. J Neurophysiol 76:1439-1456.

Mountcastle VB, Lynch JC, Georgopoulos AP, Sakata H, Acuna C (1975) Posterior parietal association cortex of the monkey: command functions for operations within extrapersonal space. J Neurophysiol 38:871-908.
Nixon PD, McDonald KR, Gough PM, Alexander IH, Passingham RE (2004) Cortico-basal ganglia pathways are essential for the recall of wellestablished visuomotor associations. Eur J Neurosci 20:3165-3178.

Pasupathy A, Miller EK (2005) Different time courses of learning-related activity in the prefrontal cortex and striatum. Nature 433:873-876.

Petrides M (1982) Motor conditional associative-learning after selective prefrontal lesions in the monkey. Behav Brain Res 5:407-413.

Petrides M (2005) Lateral prefrontal cortex: architectonic and functional organization. Philos Trans R Soc Lond B Biol Sci 360:781-795.

Powell KD, Goldberg ME (2000) Response of neurons in the lateral intraparietal area to a distractor flashed during the delay period of a memoryguided saccade. J Neurophysiol 84:301-310.

Rizzolatti G, Riggio L, Dascola I, Umilta C (1987) Reorienting attention across the horizontal and vertical meridians-evidence in favor of a premotor theory of attention. Neuropsychologia 25:31-40.

Schlag-Rey M, Amador N, Sanchez H, Schlag J (1997) Antisaccade performance predicted by neuronal activity in the supplementary eye field. $\mathrm{Na}$ ture 390:398-401.

Snyder LH, Batista AP, Andersen RA (1997) Coding of intention in the posterior parietal cortex. Nature 386:167-170.

Stoet G, Snyder LH (2004) Single neurons in posterior parietal cortex of monkeys encode cognitive set. Neuron 42:1003-1012.

Toni I, Passingham RE (1999) Prefrontal-basal ganglia pathways are involved in the learning of arbitrary visuomotor associations: a PET study. Exp Brain Res 127:19-32.

Toni I, Rushworth MFS, Passingham RE (2001) Neural correlates of visuomotor associations-spatial rules compared with arbitrary rules. Exp Brain Res 141:359-369.

Toth LJ, Assad JA (2002) Dynamic coding of behaviourally relevant stimuli in parietal cortex. Nature 415:165-168.

Wallis JD, Miller EK (2003) From rule to response: neuronal processes in the premotor and prefrontal cortex. J Neurophysiol 90:1790-1806.

Wallis JD, Anderson KC, Miller EK (2001) Single neurons in prefrontal cortex encode abstract rules. Nature 411:953-956.

White IM, Wise SP (1999) Rule-dependent neuronal activity in the prefrontal cortex. Exp Brain Res 126:315-335.

Wise SP, Boussaoud D, Johnson PB, Caminiti R (1997) Premotor and parietal cortex: corticocortical connectivity and combinatorial computations. Annu Rev Neurosci 20:25-42.

Zhang J, Riehle A, Requin J, Kornblum S (1997) Dynamics of single neuron activity in monkey primary motor cortex related to sensorimotor transformation. J Neurosci 17:2227-2246.

Zhang M, Barash S (2000) Neuronal switching of sensorimotor transformations for antisaccades. Nature 408:971-975.

Zhang M, Barash S (2004) Persistent LIP activity in memory antisaccades: working memory for a sensorimotor transformation. J Neurophysiol 91: 1424-1441. 